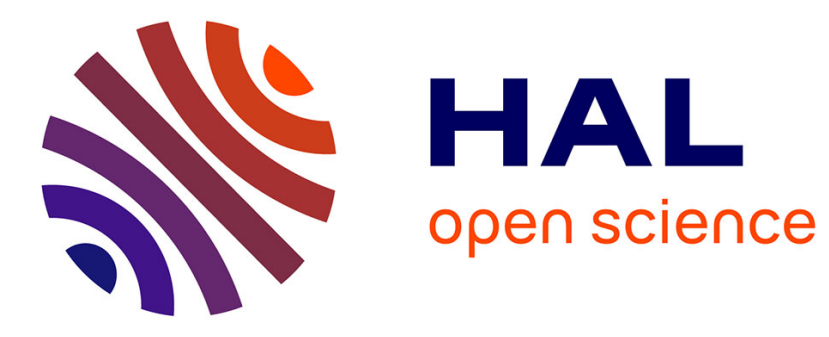

\title{
L'éducation thérapeutique en question. Topiques plurielles
}

Chantal Eymard, Christiane Peyron-Bonjan, Vincent Bonniol, Jean Michel Perez

\section{To cite this version:}

Chantal Eymard, Christiane Peyron-Bonjan, Vincent Bonniol, Jean Michel Perez. L'éducation thérapeutique en question. Topiques plurielles. Santé Décision Management, 2010, 12 (1-4), pp.115 124. 10.3166/sdm.13.115-124 . hal-01628351

\section{HAL Id: hal-01628351 https://hal-amu.archives-ouvertes.fr/hal-01628351}

Submitted on 3 Nov 2017

HAL is a multi-disciplinary open access archive for the deposit and dissemination of scientific research documents, whether they are published or not. The documents may come from teaching and research institutions in France or abroad, or from public or private research centers.
L'archive ouverte pluridisciplinaire $\mathbf{H A L}$, est destinée au dépôt et à la diffusion de documents scientifiques de niveau recherche, publiés ou non, émanant des établissements d'enseignement et de recherche français ou étrangers, des laboratoires publics ou privés. 


\section{« L’éducation thérapeutique » en question}

\section{Topiques plurielles}

\section{Chantal Eymard* - Christiane Peyron-Bonjan **- Vincent Bonniol ***- Jean Michel Perez ${ }^{* * * *}$}

* Maître de conférences HDR

UMR ADEF

Université de provence

29 avenue Robert Schuman

13320 Aix-en-provence

chantal.eymard@univ-provence.fr

** Professeur des Universités

IRSIC EA 4262 \& UMR ADEF (associée)

Université de la Méditerranée

58 boulevard C.Livon

13284 Marseille cedex 07

peyron.bonjan@univmed.fr

*** MD PhD Maître de conférences

UMR ADEF

Université de provence

29 avenue Robert Schuman

13320 Aix-en-provence

v.bonniol@free.fr

****Attaché Temporaire d'Enseignement et de Recherche

UMR ADEF

Université de provence

29 avenue Robert Schuman

13320 Aix-en-provence

jean-michel.perez@univ-provence.fr

RÉSUMÉ.

Cet article met en question la notion d'éducation thérapeutique à partir de trois modélisations théoriques : scientifique, systémique complexe et phénoménologique. 
Une première topique s'inscrit dans la constitution historique de la science médicale et montre que le concept d'éducation thérapeutique est étranger à la posture du médecin/expert. Une deuxième topique, à savoir celle du système complexe du monde de la santé, explicite que la notion d'éducation thérapeutique est plutôt de l'ordre d'une praxéologie visant des apprentissages coopératifs et récursifs. Une troisième topique phénoménologique, argumente que l'éducation thérapeutique est un leurre en raison de l'impossibilité de la communication des consciences..

ABSTRACT.

This article discusses the concept of therapeutic education from three theoretical model s: scientifical, systemical complexity, phenomenological.

A first topic part's in the historical constitution of medical science as the notion of therapeutic education is foreign to the posture of the doctor / expert. A second topic, namely the complex world of health, expressed that the concept of therapeutic education is more in the order of a praxeology for cooperative and recursive learning. A third topic, phenomenological, argues that therapeutic education is a lure because the impossibility of consciousness communication ...

MOTS-CLÉS: Modèles théoriques - Science médicale- Systémique complexe-Phénoménologie KEYWORDS : Theoretical-Models - Medical-Science- Systemical-Complexity - Phenomenology

DOI:10.3166/SDM.12.1.1-n (c) 2009 Lavoisier, Paris

\section{Introduction}

Afin de mettre en débat la notion d' « éducation thérapeutique » au lieu simplement de l'affirmer comme un «allant de soi » de la langue ordinaire, nous nous intéresserons dans cet article au décalage entre notions et concepts. Une notion est polysémique et un concept est univoque car il se réfère toujours à un modèle théorique précis. Pour l'analyse, nous avons choisi d’évoquer trois théorisations : la médecine actuelle en raison de la constitution historique de la science médicale occidentale, la systémique complexe du monde de la santé en raison de son omniprésence institutionnelle, et enfin la phénoménologie pour pouvoir dire l'éprouvé du patient. Ces modélisations feront office de mises en scènes différentes intitulées « topiques ».

\section{Topique I : la science médicale}

Les premières traces écrites de médecine remontent au 18e siècle avant JC. L'Ecole de médecine d'Hippocrate le Grand (460-370 avant JC) a permis en Grèce antique d'instituer cet art médical comme une discipline à part entière. A Alexandrie, Hérophile (300-200 avant JC) disséquait très régulièrement des 
cadavres humains car cela était autorisé dans cette ville. Malheureusement, ses nombreux écrits et dessins ont été en grande partie perdus dans l'incendie de la grande bibliothèque. La notion de diagnostic médical existe depuis ces époques et ces textes. On peut donc penser avec Galien (131-216 après JC) que « le meilleur médecin est aussi philosophe ».

Comme dans la philosophique hellénique tout corps était partie prenante du cosmos, comprendre ce dernier permettait de se connaître et de mieux vivre car, en pur respect de la vision du monde hellénique, le bien être était visé pour tout être humain. Or, penser, réfléchir, somme toute philosopher sur l'Etre en tant qu'Etre suffisait !... D'ailleurs, les expériences pratiques médicales de l'époque tentaient toujours de rétablir l'équilibre face à quelque déséquilibre que ce soit afin de vivre en convenance avec l'Etre originaire. Et compte tenu des moyens et des connaissances de l'époque c'était préférable !

Puis advint la Renaissance. L'humanisme fut adjoint à la conception moderne des sciences ; conception irriguée par un mode de pensée analytique de la méthode cartésienne. Et les médecins continuèrent à disséquer des corps, à les découper en vue de mieux connaître, mieux comprendre chaque organe et chaque fonction. Au cours du XXème siècle dernier et en raison des avancées majeures de la médecine moderne, le médecin devint technicien puis ingénieur de santé. La science et ses lois le maintiennent aujourd'hui dans la distanciation vis-à-vis du malade et de la maladie. S'il reste « clinicien » le médecin, trop impliqué dans la relation avec son patient, perd de son pouvoir de résolution de problèmes : diagnostic et thérapeutique en pâtissent. Le médecin, humaniste par nature, doit se cacher du malade et de la maladie pour survivre aux malades, à leurs maladies et faire vivre son génie et son art, fût-ce dans sa propre souffrance physique et mentale comme certains médecins héros modernes bien connus... De nos jours, le médecin ne choisit pas cette distanciation mais PROPOSE des démarches diagnostiques et thérapeutiques. Il vise tout autant le respect du patient, que la promotion de la santé : la construction entre experts thérapeutes et malades de leur « prise en charge » par le monde de la santé.

La science médicale contemporaine est celle du corps et de ses pulsions, de ses besoins et de ses déséquilibres, voire de ses errements. Diagnostics et thérapies ne réclament que distanciation et technicité. La science médicale n’est ni une science de l'implication ni une science de l'Education. Le professionnel de santé ne discute pas de l'organisation de la maison et de la famille. Le serment d'Hippocrate exige qu'il taise ce qu'il aura entendu vu ou compris dans les maisons où il sera introduit. Sa sphère n'est pas celle des pratiques humaines qu'il faudrait infléchir ou modifier en fonction de connaissances acquises. Sans distinction d'origine ou de croyance, entre le pauvre et le riche, le faible ou le puissant, le médecin doit prendre en charge le patient comme il vient sans chercher à le transformer en un clone "parfait". Le respect de la vie, le respect du patient passe par cette posture déjà parfois assez intrusive physiquement. La démarche clinique, centrée sur la plainte, sur la demande 
du patient, ou de sa famille, demeure au contact de ces derniers mais en ciblant surtout la séméiologie en vue du diagnostic et en visant une remédiation.

Le métier de médecin/soignant n'est vraiment pas celui d'éducateur : il n'en partage pas les modèles (comment faire passer les savoirs ou les bonnes pratiques ?). Il est toujours attaché à une déontologie, à des choix éthiques, à son art et aujourd'hui à sa science, mais le moins possible à son patient. Cette science médicale ne peut être celle de l'éducateur. Son éthique, tout en demeurant respectueuse des libertés individuelles du patient, demeure toujours celle du bios, de l'urgence vitale - donc jamais celle soupesée et réfléchie voire intellectuelle de l'éducateur. La science est celle, froide, qui exige de voir mourir les malades quotidiennement sans défaillir et en cherchant toujours à les retenir, et à comprendre ce qui les tue !

Ce métier ne repose pas non plus sur les fondements de l'instruction (que transmettre ?). Il n'en partage pas la philosophie car les acteurs de santé ne sont pas des répétiteurs. Leur indépendance historique est fondamentale, et leur interdit presque tout asservissement à un corpus de pensées et de pratiques. Leur Art ne doit s'appuyer que sur LEUR connaissance et LEUR intime conviction pour pratiquer ce qu'il y a de meilleur à chaque instant envers son patient. Leur ordre, leur guilde ou leur corporatisme les a toujours tenus à l'écart de tout pouvoir.

Le contrat qui se noue entre chaque professionnel de santé et son patient n'est pas un contrat de formation ou d'éducation. Ce contrat s'établit sur le colloque singulier, sur la confiance et l'absence de jugement, sur le secret ; le praticien doit à son patient les meilleurs soins, la meilleure prise en charge diagnostique et thérapeutique, dans les règles de son Art et en toute indépendance. Il ne pourra être jugé là-dessus que par ses pairs. Cet art et cette indépendance reposent aujourd'hui plus que jamais sur les expérimentations, les preuves et l'évaluation des pratiques. Science, thérapie et éducation sont étrangers. Alors, pourrait-on comprendre autrement la notion d'éducation thérapeutique puisque c'est un non sens lorsque l'on raisonne à partir de la science médicale ? Faudrait-il pour cela abandonner la topique de cette expertise pour une autre, à savoir celle du système complexe configuré par tous les acteurs du monde de la santé ?

\section{Topique II : la systémique complexe}

Si le soigné se devait dans la première topique d'obéir aux prescriptions de l'expert car il savait qu'il ne savait rien... peut-il être " éduqué » grâce à toutes les parties prenantes de l'organisation systémique du monde de la santé ; ces parties prenantes étant entendues en un sens large : médecins, soignants, psychologues, sociologues, travailleurs sociaux, associations de patients, patients.... ? Si l'on se réfère à la première étymologie latine du verbe éduquer, à savoir educare, on peut certes « élever » les patients en leur explicitant les diagnostics, les conseils et les thérapies choisies par les professionnels, les soignés renforçant les bienfaits des 
médications, des traitements, des consignes de bonne santé et de vie plus saine en les comprenant et en les opérant plus correctement. Cependant la notion d'éducation thérapeutique est alors plutôt une praxéologie, entendue comme l'aide apportée aux patients, à leurs familles et/ou leur entourage pour « comprendre la maladie et les traitements, collaborer aux soins, prendre en charge leur état de santé et conserver et/ou améliorer [leur] qualité de vie» (Ministère de la santé, 2007).

Cette praxéologie multiprofessionnelle et pluridisciplinaire se rangerait donc plutôt sous l'égide des théories de l'apprentissage. Selon le modèle de l'apprendre dominant, la finalité de cette théorie de l'action vise davantage une modification, soit du comportement, soit du processus de pensée et met l'accent sur l'apprentissage systémique du patient (Deccache, 2000). Or, les théories de l'apprentissage sont traditionnellement sous-tendues par deux types de modèles théoriques :

- Les premiers privilégient une approche behavioriste ou cognitivocomportementaliste de l'apprendre.

L'ancrage behavioriste respecte les principes suivants :

- $\quad$ «tout apprentissage est observable par le changement de comportements qu'il implique ;

- $\quad$ les savoirs acquis sont cumulatifs ;

- $\quad$ la réussite étant un facteur de motivation, il est important de la renforcer ;

- $\quad$ les savoirs complexes doivent être décomposés en savoirs élémentaires ;

- $\quad$ les objectifs à atteindre doivent être progressifs pour favoriser la réussite ;

- les exercices d'applications favorisent la généralisation et la maîtrise des acquisitions. » (Eymard, 2010, p.26)

L'ancrage cognitivo-comportementaliste tend à œuvrer pour l'obtention de comportements adaptés aux problèmes de santé. Il est alors attendu du soigné qu'il développe sa motivation à apprendre, adopte des représentations en matière de santé et de maladie conformes aux normes scientifiques, efface ses croyances et pensées dysfonctionnelles, et gère au mieux ses états émotionnels (Bury \& Foucaud, 2009).

Pour le système de santé la réussite des apprentissages constitue alors un des enjeux majeurs car elle a pour fonction de remplir une sorte de contrat politique de toutes les parties prenantes du système sanitaire. Ce contrat tissé entre les professionnels du monde de la santé et les patients ne vise qu'un objectif : faire advenir le modèle précis d'un corps et d'un esprit sain décrits par les sciences les plus avancées. Avec cette définition de la notion d'éducation thérapeutique sous l'égide de la première étymologie, on s'inscrit plutôt dans un mode de pensée linéaire et causaliste : des causes de dysfonctionnements de telle ou telle partie du corps (voire de la psyché), des moyens de les éradiquer et d'une possible adaptation de ces moyens grâce à une communication efficiente soignants-soignés.

Les expériences classiques d'éducation thérapeutique sont souvent entendues en ce sens à savoir celui d'un apprentissage à un "vivre en santé », apprentissage 
confronté à un rapport au temps (vie/mort de la maladie, de sa chronicité). On comprend bien dès lors, que lorsque les pratiques soignantes se réfèrent (consciemment ou inconsciemment) aux modèles behavioriste ou cognitivocomportementaliste de l'apprentissage, le rapport aux savoirs des professionnels de la santé risque de glisser vers une posture plutôt dogmatique en voulant instruire les patients qu'ils imaginent $a$ priori comme ignorants des normes de «bonne santé ».

\section{La notion d'، éducation thérapeutique " instruit, régule mais n'éduque point.}

- Les seconds modèles sont constructivistes ou socio-constructivistes.

Avec les travaux de Bruner (1963), l'accent est mis sur le processus de pensée de l'apprenant et en particulier sur sa possibilité d' « apprendre à apprendre ». Lorsque les soignants incitent les soignés à développer des analyses de leurs apprentissages, ils privilégient leur métacognition et enrichissent en raison de cela le processus de pensée des soignés. Les textes de Vygotski (1934) prennent en compte le contexte environnemental et invitent à une fonction de médiation pour favoriser les apprentissages. La communauté de patients peut alors être sollicitée pour venir témoigner des apprentissages réalisés dans la conformité aux « bonnes normes de santé » et expliciter la qualité de vie atteinte grâce à cela. Considéré comme interdépendant du contexte dans lequel il se construit, tout apprentissage délibéré privilégie l'expérience du patient ou du groupe de patients, ainsi que sa capacité à expliciter son action et à formaliser les apprentissages réalisés. Dans cette dynamique, les patients peuvent aussi co-inventer avec les soignants un traitement plus en conformité à leurs desiderata, leurs modes de vie, leurs cultures...Le soignant, en tant qu'organisateur des situations d'apprentissage " va donc avoir à gérer de l'hétérogène, avoir le souci de permettre à chacun d'emprunter sa propre voie ; chacun se développera " grâce à », « avec » et même « contre » sa rencontre et sa confrontation avec les autres dans le cadre des interactions qui parcourent la situation » (Genthon, 1996, p.63). Le système de santé attend alors des patients qu'ils interagissent eux-mêmes sur leur environnement pour que ce dernier devienne le plus favorable à leur bien-être en santé.

Avec la théorie de l’apprentissage social (Bandura, 1977), quatre principes peuvent être retenus dans les programmes mis en place en éducation thérapeutique : les individus peuvent apprendre par l'observation du comportement et des résultats des autres ; Cette expérience de subsitution (apprentissage vicariant) contribue à l'acquisition de savoir-faire sans passer obligatoirement par un processus progressif d'essais et erreurs. Il ne dispense pas de l'expérience directe ; les cognitions jouent un rôle fondamental; les individus ont davantage tendance à imiter un comportement s'ils en perçoivent des renforcements positifs. A partir de cette théorie, Bandura (1986), développe la théorie sociale cognitive, dans laquelle la notion d'auto-efficacité ou de sentiment d'efficacité personnelle est considérée comme la base de la motivation du sujet et de sa persévérance dans l'effort pour apprendre. 


\begin{abstract}
L'apprentissage coopératif et le conflit socio-cognitif permettent aux patients non seulement d'approprier des savoirs précis, mais aussi de s'adapter à leur environnement grâce à une récursivité théorie-pratique. Le soignant devient un organisateur de situations favorables au processus d'apprentissage des patients entre eux. Le défi est alors de susciter leurs analyses critiques, et aussi leurs compétences psychosociales, comme aptitudes à développer un état de bienêtre mental, en adoptant des comportements appropriés à l'occasion des interrelations. Les soignés sont invités à être acteurs de leur santé et de leur qualité de vie dans une perspective d'empowerment (Rapaport, 1984).
\end{abstract}

Dans cette perspective socio-contructiviste, le contrat entre les parties prenantes du système sanitaire devient socio-politique. Il a pour finalité, non seulement de permettre aux soignés de devenir acteurs coopératifs de leur «bonne santé », mais aussi d'acquérir des qualités psycho-sociales telles que «l'estime » (Coopersmith, 1999), la « confiance le « sentiment d'efficacité » (Bandura, 1986). Ainsi, ils seront les acteurs d'une société viable.

Comme la première mise en scène, (à savoir celle de la science médicale) a montré l'impossibilité de confondre le concept d'éducation avec celui de science, comme la deuxième mise en scène, (à savoir celle du système complexe de santé) a explicité que la notion d'éducation thérapeutique n'était qu'une praxéologie conduite en vue d'apprentissages divers, est-il possible d'entendre autrement l'éduquer? La langue latine, nous autorise à considérer une autre étymologie « $e$ ducere » c'est à dire « conduire hors de soi ». Serait-il alors permis aux patients de sortir de l'éprouvé douloureux de leur maladie incrustée dans leur chair et/ou dans leur esprit? Les patients pourraient-ils être incités à se projeter vers un devenir possible ? Si oui comment? Avant de répondre à ces questions, il est nécessaire de rappeler que les deux premières topiques reposaient sur la philosophie de la représentation instituant le concept de sujet face au monde (qu'il soit médecin expert ou patient interagissant dans le système complexe de santé). Or, pour tenter de comprendre l'éprouvé d'un patient, pour atteindre sa conscience interne, il est nécessaire de rompre avec la philosophie traditionnelle et d'entrer dans une vision du monde phénoménologique. Seule cette mise en scène permet d'entendre son cœur précieux : la conscience interne (le cogito). Pour ce faire cette philosophie met entre parenthèses le sujet et le monde.

\title{
4. Topique III : la phénoménologie
}

Husserl décrit les contenus et les significations qui apparaissent dans la conscience. Il abolit donc la distance entre le sujet qui apprend ou connaît et ce qui est appris ou connu. « Par l'intentionnalité, la conscience est une activité donatrice 
de sens, elle ne reçoit pas un objet tout constitué mais elle en constitue le sens par le mouvement même par lequel elle se dirige vers lui. L'intentionnalité est cette activité par laquelle une conscience se pose comme contenu de pensée ou noèse et pose par là même un objet pensé ou noème. La conscience naïve croyait recevoir un monde tout constitué alors qu'elle en était la source fondatrice » (Peyron-Bonjan, 2010). Le fait que la conscience soit activité garantit le primat de la conscience sur l'objet. Par l'épochè phénoménologique ${ }^{5}$, nous suspendons toute adhésion naïve au monde et au sujet et atteignons la conscience...

L'héritier de Husserl, Heidegger institue les concepts de Dasein (être-le-là) et celui de Sein zum zeit (être-pour-la-mort). Pour cet auteur, seuls les existentiaux permettent de montrer les expérientiels des sujets; seul le Dasein manifeste son authenticité dans l'angoisse car l'expression de l'existence déchue permet d'atteindre le troisième existential ou souci. Comme le passé est oubli de l'Etre et comme le présent est anonyme, seul l'avenir peut exprimer la transcendance du sujet. L’Etre est immédiatement rapporté au Temps (Heidegger, 1927). L'anticipation de soi et le pouvoir être ne seraient révélés que par la maladie ; d'où la conscience active de la vacuité de toutes les actions et l'amour du destin. Seules la maladie et la mort donnent un sens au Dasein : « de la certitude d'être enfin arrivé là et d'avoir rattrapé son destin, dédouané de toute quête,[...] mille détours sont possibles, mille traverses, mille impasses, mille esquives et tentatives de dire cette énigme ravivée que [je] suis, que [je] deviens ». (Deschamps, 2002, p.111).

En respectant d'une part les concepts clefs des auteurs phénoménologues et d'autre part la deuxième étymologie latine du verbe éduquer on pourrait penser l'expression «e-ducere » c'est à dire « conduire hors de soi » comme une incitation pour chaque patient à se projeter vers son devenir, mais pourrait on encore parler d' « éducation thérapeutique » sous cet angle de vue ? Non, car toute mise en scène phénoménologique met en question l'intersubjectivité (Husserl, 1929) et aucun professionnel de santé ne peut avoir accès au processus interne de la conscience d'autrui ; a fortiori, aucun expert n'a connaissance de ce qu'autrui a comme impression intime de la "vie saine» et du «bien être». L'éprouvé de la " sanitude », de l'équilibre, est propre à chacun et diffère à chaque instant. La statue du Commandeur de la bio-médecine techniciste est alors fort loin... et donc toute notion de remédiation s'éloigne. Chaque conscience inventant son équilibre à l'intérieur de ses déséquilibres et ses thérapies propres (dans sa manière d'être-aumonde ici et maintenant), voire même en refusant toute thérapie extérieure...

Tout mode de pensée "scientiste » présupposait un savoir-pouvoir (Foucault, 1963) et les conseils thérapeutiques le renforçaient; de patient subissant les traitements, il devenait patient apprenant. Mais si l'on privilégie l'éprouvé - en un sens phénoménologique - et si on le met au centre de la réflexion du processus de

\footnotetext{
${ }^{5}$ Mise entre parenthèses du Sujet et du Monde rendue nécessaire afin de ne pas demeurer dans une philosophie de la représentation, philosophie séparant le sujet du Monde.
} 
vie de chaque conscience singulière, l'éducation est une aporie car aucun expert, ( $1^{\text {ère }}$ topique) ni aucun système de santé ( ${ }^{\text {ème }}$ topique), n'a accès ni à la singularité de chaque conscience ni à l'image que cette dernière aurait du corps en bonne santé hic et nunc (ici et maintenant). Et toute éducation dite thérapeutique serait un leurre même en considérant les témoignages singuliers de l'expérientiel de patients vers d'autres patients ; car ces récits peuvent ou pas déclencher quelques prises de conscience interne

d'autres patients. Ce ne peut être qu'un pari, un espoir...

- pour qu'ils ressentent autrement leur vécu ou pas,

- pour qu'ils transcendent tout autant les thérapies que l'environnement du système de soins ou pas,

- $\quad$ pour qu'ils vivent au mieux ou pas...

Dans cette mise en scène, le professionnel de santé n'a pas à intervenir davantage si ce n'est que pour témoigner de sa place, de son expérience. Sa posture hiérarchique n’est absolument pas légitime.

\section{Conclusion}

Force est de constater que la notion d'«éducation thérapeutique » est trop souvent ancrée dans un modèle de l'expertise, de l'apprentissage des normes de « bonne santé » au détriment du processus de vie de chaque sujet singulier. Les deux premières topiques pourraient donc être parfois synonymes de violence institutionnelle dans leur refus de l'altérité ce qui risque de renforcer les « résistances des patients à tenir compte des messages hétéro-nomes des soignants » (Lecorps \& Paturet, 1999, p.147)... et à se soigner !... « La science moderne et son idéal d'objectivation nous imposent à nous tous, que nous soyons médecins ou patients ou encore simples citoyens attentifs et vigilants le risque d'une très forte aliénation» (Gadamer, 1993, p.81).

Donc, la santé ne peut plus relever uniquement du secteur sanitaire : elle dépasse la normalisation d'une vie saine pour viser le bien être individuel. La santé est « pour chacun, tout au long de la vie, un mode de présence au monde : joie et performance tout autant que confrontation à la douleur, à la souffrance et à la mort. La santé est à la fois le processus indéfini de l'expression de soi et dans ce même processus, expérience de la limite, du vieillissement, du handicap parfois, de la maladie et glissement vers la mort. » (Lecorps, 2008, p.2).

\section{Bibliographie}

Bandura, A. Self-efficacy,1986, in Bandura (ed) Social foundation of thought \& Action: Asocial cognitive theory: Prentice Hall. 
$10 \mathrm{SDM}-12 / 2009$.

Bury J. A., Foucaud J. L’éducation thérapeutique In : F. Bourdillon dir. Traité de prévention. Paris : Flammarion Médecinesciences, coll. Traités, 2009, p. 81-87.

Bruner J.S. The process of education, Vintage Books. Newyork, 1962, 97 p.

Coopersmith SEI. Inventaire d'estime de soi. Revue Européenne de Psychologie Appliquée, 1999.

Deccache A. Quelles pratiques et compétences en éducation du patient : Recommandations de l'OMS. Bulletin d'éducation du patient, vol.19, n¹, 2000.

Deschamps D. Psychanalyse et cancer, au fil des mots... un autre regard. Paris. L'Harmattan, 2002.

Eymard C. Des modèles de l'éducation et de la santé à l'activité d'éducation thérapeutique. In, Foucaud J., Balcou-Debussche M., Bury J., Eymard C. (sous la dir.) Éducation thérapeutique du patient : modèles, pratiques et évaluation. Saint-Denis : INPES, coll. Santé en action, 2010.

Foucault, M. Naissance de la clinique : une archéologie du regard médical. Paris : PUF, 1963.

Gadamer H-G. Philosophie de la santé, (trad. M. Dautrey). Paris : Grasset, 1993.

Genthon M. Apprentissage-Evaluation-Recherche. Genèse des interactions complexes comme ouvertures régulatrices. En question, Université de Provence, 1997.

Heidegger M. Etre et Temps. Paris : Gallimard, 1927 (1964).

Husserl E. Méditations cartésiennes. Traducteurs E. Levinas G.Peiffer, 1929.

Lecorps Ph. Les enjeux d'une prévention prévenante. Rouen : Source : www.ac-rouen.fr 23/04/08, 2008, $16 \mathrm{p}$.

Lecorps Ph. \& Paturet J-B. Santé publique, Du biopouvoir à la démocratie. ENSP, 1999.

Ministère de la Santé. Plan pour l'amélioration de la qualité de vie des personnes atteintes de maladies chroniques et des Solidarités. (2007-2011). Avril 2007. En ligne : www. santejeunesse-sports.gouv.fr/dossiers/ sante/maladies-chroniques/plan-pourameliorationqualite-vie-personnes-atteintesmaladies-chroniques-2007-2011.htm [dernière consultation le 1er Juillet, 2010]

Peyron-Bonjan, C. Le cercle des concepts disparus. Paris : coll AFIRSE, ed l'Harmattan, 2010, 280 p.

Rappaport J. Studies in Empowerment : introduction to the Issues. Prevention in Human Services. $1984, n^{\circ} 3$ : p. 1-17.

Vygotskij L-S. Pensée et langage. Paris :Messidor : Éd. sociales, coll. Terrains, 1985, 419 p. (1934) 